\title{
Revisiting optimal needle size for thyroid fine-needle aspiration cytology: not much finer, less non-diagnostic?
}

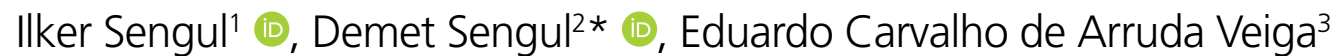

Optimal needle size for thyroid fine-needle aspiration (FNA) cytology has not been established distinctly and conclusively in thyroidology to-date. The literature on the subject is scarce. We read with respect the research article, entitled: "Optimal needle size for thyroid fine-needle aspiration cytology ${ }^{1}$." Tanaka et al. ${ }^{1}$ declared to utilize two different sizes, 22- and 25-gauges, of needles for the FNA procedures. The authors reported the nondiagnostic/unsatisfactory rates of 22- and 25-gauge needles were being as $18.5 \%$ and $21.0 \%$, respectively. Nevertheless, we currently reported the possible efficacy of nodule size of 10 - and $15-\mathrm{mm}$ in the greatest dimension, as the cutoff points, on three diagnostic tools; (i) strain elastography (SE), (ii) ultrasonography-guided FNA (US-FNA) cytology, and (iii) histopathology ${ }^{2}$. We had utilized just 27-gauge fine-needle (Hayat, $2 \mathrm{ml} 3$ P 27-G, $0.40 \times 50$ mm, Istanbul, Turkey) by performing US-FNA for a total of 425 cases with 500 thyroid nodules by the surgeon-performed ultrasonography (SUS), based on the revised American Thyroid Association (ATA) management guidelines for patients with thyroid nodules and differentiated thyroid cancer [i.e. low, intermediate, and high suspicion nodules; 2015 ATA management guidelines for adult patients with thyroid nodules and differentiated thyroid cancer] for the duration of 3 years and 2 months ${ }^{2,3}$. This is a considerable and novel 'SUSbased' US-FNA thyroid study for the specific and also 'well-accepted crucial' size cutoffs of 10 - and $15-\mathrm{mm}$ in endocrine surgery, endocrine pathology, and thyroidology, to-date ${ }^{2}$. To this end, the cytopathologic evaluation of the cases had been performed based on The Bethesda System for Reporting Thyroid Cytopathology (TBSRTC), 1st ed. ${ }^{4}$ and our FNA cytology outcomes for nondiagnostic/unsatisfactory cytology were revealed as $9.0 \%{ }^{2}$. Moss et $\mathrm{al}^{5}$ reported in a systematic review and meta-analysis that the regular and coordinated thyroid FNA should be performed with smaller needle gauges, 24-27-gauges, without aspiration, routinely. In addition, we very recently introduced and suggested a novel terminology, in this sense, termed as "minimally invasive FNA; MIFNA" and "minimally invasive thyroid FNA; Thy MIFNA" involving 27-gauge fine-needle with topical and local anesthetic agents administration with the reasonable rates of nondiagnostic cytology, TBSRTC ${ }^{6,7}$.

Of note, we recommend opting for Thy MIFNA with 27-gauge fine-needle to practice US-FNA procedure for indicated suspicious thyroid nodules with reasonable low rates of Category I (TBSRTC, 1 st and 2 nd eds.) and probably low severity of pain. To this end, we also recommend wielding facilitating pre- or periprocedural local, even topical anesthetic agents toward US-FNA for the thyroid nodules ${ }^{2,6,7}$. As a matter of fact, this issue merits further investigation.

\section{ACKNOWLEDGMENTS}

We thank all of the article participants.

\section{AUTHORS" CONTRIBUTIONS}

IS: Conceptualization, Data curation, Formal analysis, Investigation, Methodology, Project administration, Resources, Software, Validation, Visualization, Writing - original draft, Writing - review \& editing. DS: Conceptualization, Data curation, Formal analysis, Investigation, Methodology, Project administration, Resources, Software, Supervision, Validation, Visualization, Writing - original draft, Writing - review \& editing. ECAV: Investigation, Methodology, Project administration, Supervision, Visualization, Writing - review \& editing.

\footnotetext{
${ }^{1}$ Giresun University, Faculty of Medicine, Division of Endocrine Surgery, Department of General Surgery - Giresun, Turkey.

${ }^{2}$ Giresun University, Faculty of Medicine, Department of Pathology - Giresun, Turkey.

3Universidade de São Paulo, Faculdade de Medicina de Ribeirão Preto, Departamento de Obstetricia e Ginecologia - São Paulo (SP), Brazil.

*Corresponding author: demet.sengul.52@gmail.com

Conflicts of interest: the authors declare there is no conflicts of interest. Funding: none.

Received on July 14, 2021. Accepted on July 18, 2021.
} 


\section{REFERENCES}

1. Tanaka A, Hirokawa M, Higuchi M, Kanematsu R, Suzuki A, Kuma $S$, et al. Optimal needle size for thyroid fine needle aspiration cytology. Endocr J. 2019;66(2):143-7. https://doi. org/10.1507/endocrj.EJ18-0422

2. Sengul D, Sengul I, Egrioglu E, Ozturk T, Aydın I, Kesicioglu T, et al. Can cut-off points of 10 and $15 \mathrm{~mm}$ of thyroid nodule predict malignancy on the basis of three diagnostic tools: i) strain elastography, ii) the Bethesda System for Reporting Thyroid Cytology with 27-gauge fine-needle, and iii) histopathology? J BUON. 2020;25(2):1122-9. PMID: 32521915

3. Haugen BR, Alexander EK, Bible KC, Doherty GM, Mandel SJ, Nikiforov YE, et al. 2015 American Thyroid Association Management Guidelines for Adult Patients with Thyroid Nodules and Differentiated Thyroid Cancer: The American Thyroid Association Guidelines Task Force on Thyroid Nodules and Differentiated Thyroid Cancer. Thyroid. 2016;26(1):1-133. https://doi.org/10.1089/thy.2015.0020
4. Cibas ES, Ali SZ. The Bethesda system for reporting thyroid cytopathology. Thyroid. 2009;19(11):1159-65. https://doi. org/10.1089/thy.2009.0274

5. Moss WJ, Finegersh A, Pang J, Califano JA, Coffey CS, Orosco RK, et al. Needle biopsy of routine thyroid nodules should be performed using a capillary action technique with 24- to 27-gauge needles: a systematic review and metaanalysis. Thyroid. 2018;28(7):857-63. https://doi.org/10.1089/ thy. 2017.0643

6. Sengul I, Sengul D. Proposal of a novel terminology: minimally invasive FNA and thyroid minimally invasive FNA; MIFNA and thyroid MIFNA. Ann Ital Chir. 2021;92:330-1. PMID: 34312332

7. Sengul I, Sengul D. Delicate needle with the finest gauge for a butterfly gland, the thyroid: is it worth mentioning? Sanamed. 2021;16(2):173-4. https://doi.org/10.24125/ sanamed.v16i2.515 